\title{
AVERROES COMO MUFTÍ
}

\author{
Juan Martos Quesada \\ Universidad Complutense
}

\section{RESUMEN}

El artículo trata de la actividad de Averroes como muftí, del papel que juega esta figura jurídica en el mundo del Derecho andalusí, de la confusión entre Averroes y su abuelo acerca de la donación de fetuas $\mathrm{y}$, por último, en el carácter jurista de Averroes.

Palabras clave: muftí, Derecho, fiqh, fetua.

\begin{abstract}
This paper is about the activity of Averroes as mufti, the rol of this occupation in the legal world in al-Andalus; also, about the confusión between Averroes and his grandfather in the donation of fetuas and, finally, the legal features of Averroes.
\end{abstract}

Key words: mufti, law, fetua.

\section{EL AVERROES JURISTA: PRINCIPALES ESTUDIOS DESDE ESTA PERSPECTIVA}

La relevancia del carácter polifacético de la actividad intelectual y científica de Averroes, es uno de los logros más visibles del esfuerzo, lento y tenaz, de los actuales investigadores de esta figura cumbre de la intelectualidad humana en la época medieval, posibilitando una visión de su obra y que hacer desde diversos puntos de vista y desde múltiples enfoques.

Efectivamente, desde los meritorios, pero ya obsoletos trabajos de Munk o Renan ${ }^{1}$ de finales del siglo pasado, la atención de los eruditos se había centrado en el análisis de los aspectos religiosos y teológicos de su obra, en la dirección de sus ideas científicas y, especialmente, en el estudio de sus concepciones filosóficas.

Hay que esperar a los años 60 y 70 para que autores como R. Brunschvig o A.M. Turki saquen a la luz los aspectos jurídicos de la vida y la obra de Averroes, consiguiendo con ello una visión menos injustamente fragmentaria de esta personalidad tan rica y compleja.

Una ojeada al campo bibliográfico de los estudios acerca de la labor jurídica de Averroes durante este siglo, apenas llega a la decena de trabajos dignos de mencionar. Veamoslos, pues.

Basándose en la obra de Renan, y sin cuestionarse los errores en la atribución de las obras de Averroes que ésta contiene, C.A. Nallino publica, a principios de siglo, un artículo titulado «Intorno al-Kitab al-Bajan del giurista Ibn Rushd», publicado en Homenaje a Codera, (Zara-

1 MUNK, Melanges de philosophie juive et arabe, (París, 1859), págs. 418-58. E. RENAN, Averroès et le averroüsme, (París, 1852). 
goza, 1904), al que siguen, en la misma línea errónea, los artículos de F.Viré, «Averroes juriste», en Revue Tunisienne de Droit, III (1955), págs. 28-37; y R. De Mendizábal, «Averroes, filósofo y juez», en Revista General de Legislación y Jurisprudencia, XIIV (1962), pp. 489. 533, al que le sigue «Crónica de los jueces musulmanes. III. Estirpe de juristas, los Banu Rush», publicado en la revista África, XXIII (1971), págs. 6-9.

Hay que esperar a principios de los años 60 para que R. Brunschvig escriba su importante trabajo «Averroès juriste», que vé la luz en Études d'Orient dédiées à la mémoire de LévyProvençal, (París, 1962), I, págs. 35-68, en el que reinvindica el estudio de la obra jurídica averroísta como ejemplo de aplicación metódica de los uṣul al-fiqh, a la vez que hermenéutica, en donde la objetividad, la lógica, la racionalidad y la sistematización hacen de la Bidâya -cuyo análisis conforma el núcleo del artículo- una de las obras más emblemáticas del pensamiento jurídico teórico islámico.

En la misma dirección de resaltar los aspectos teóricos del Averroes jurista, hay que mencionar los trabajos de Guennun, «Averroes, el jurista», publicado en la revista Pensamiento, XXV (1969), págs. 195-205; y M. Kassem, «La notion de justice en Islam... le concept de justice chèz Averroès», en Diogène, LXXIX (1972), págs. 81-107.

Un nuevo paso importante en el campo de estos estudios se da con el artículo de 'A.M.Turki, «La place d'Averroès juriste dans l'histoire du malikisme et de l'Espagne musulmane», recogido en la miscelánea Multiple Averroès. Actes du colloque du 850è anniversaire de la naissance d'Averroès, París, 20-23 Septiembre 1976, (París, 1978), págs. 33-43 en donde encuadra la labor jurídica de Averroes en su marco socio-histórico.

Y, por último, mencionaremos el artículo de J.Langhade y D.Mallet, «Droit et philosophie au XIIè siècle dans l'Andalus: Averroès (Ibn Rušd)», publicado en R.O.M.M., XI (1985), págs. 103-121, como la última muestra de los estudios averroístas en este campo concreto.

Prácticamente toda la bibliografía que hemos reseñado, parte del principio de la obra jurídica de Averroes como un sólido entramado arquitectónico teórico-filosófico, ignorando sus aspectos más pragmáticos; visión a la que, sin duda, ha contribuido la confusión que, sobre su autoría, ha rodeado hasta hace poco su obra.

Como ha afirmado más de un experto, el problema de la obra jurídica de Ibn Rušd es extremadamente complejo y su aclaración exige un estudio detallado de todas las obras sobre fiqh atribuidas a los Bañ Rušd.

La revisión de los manuscritos y la lectura crítica de los cataloguistas, ha resuelto definitivamente que la única obra de carácter jurídico de Averroes es la Bidāya $a^{2}$, mientras que el Kitāb Bayān al-tahsịll (Libro explicativo de la adquisición del saber jurídico comentando los Masā'il de al-'Utbi), Al-Muqaddammät (Introducciones que falicitan la comprensión de las cuestiones de jurisprudencia del derecho islámico), y el Kitāb al-nawāzil fi-l-fatāwà (Colección de sentencias y consultas jurídicas), pertenecen sin duda alguna a su abuelo Abū-l-Walīd Muḥammad Ibn Rušd; siendo errónea la atribución durante mucho tiempo hecha a su nieto, el Averroes por todos conocido.

Mientras duró la confusión, los estudiosos dieron por hecho su papel de muftí (ya que las obras recogen un sin número de fetuas) junto al de cadí, y en consecuencia, su ejercicio del derecho práctico. Pero la clarificación en la autoría de sus obras, eliminó prácticamente la figura de Averroes como jurista activo, para dar lugar a una imagen de jurista filósofo, racionalista y teórico, más en consonancia con su obra jurídica conocida, la Bidāya.

No obstante -y sin negar su importancia como estudioso del pensamiento jurídico más teórico-, intentaremos en este trabajo poner de relieve el carácter que, sin duda, tuvo como

2 Bidāyat al-muŷtahid; hay numerosas reediciones, a partir de la primera edición de El Cairo, 1909, y plagios; la más recomendable podría ser la editada en El Cairo, 1935. Una excelente traducción es la versión inglesa llevada a cabo por I.A.K. Nyazee y revisada por M. Abdul Rauf, 2 vols., Londres, 1994. 
jurista práctico, expedidor de respuestas, sentencias y soluciones a consultas jurídicas de la gente o de las autoridades jurídicas y políticas, centrándonos en su papel como muftí, a la luz de los datos que hemos analizado de su vida y su obra.

Pero, antes, estimamos pertinente hablar, aunque de forma somera de la figura del muftí, de su importante papel en al-Andalus y de su evolución en la época de Averroes.

\section{LA ACTIVIDAD JURÍDICA Y EL CARÁCTER PRÁCTICO DEL PENSAMIENTO JURÍDICO EN AL-ANDALUS: LA FIGURA DEL MUFTÍ}

Es sabido que el ejercicio del poder judicial en el Islam medieval se confunde con el ejercicio del poder legislativo. Esta fusión del poder legislativo y judicial encuentra su justificación en la misma evolución histórica del Islam: la acumulación, en los primeros tiempos del Islam, en la persona del califa del poder legislativo, ejecutivo y judicial, sufre pronto la diferenciación entre el ejecutivo, personificado por el califa, y el judicial, en la figura del cadí; los agentes subalternos de una y otra institución están claramente delimitados y, generalmente, no se confunden nunca.

Pero, por el contrario, las funciones legislativas y judiciales permanecen con una delimitación muy confusa, a lo que contribuye la amplia capacidad de poder que llega a poseer el cadí y el principio básico de que todas las leyes ya se hallan contenidas en el Corán y la sunna.

La necesidad de interpretación de estas leyes, para su aplicación a casos concretos, segregar en la función del cadiazgo una faceta de estudio y profundización, que precise la Ley y remedie el problema de sus silencios y lagunas. El juez solo no podía abarcar este campo, con lo que la figura del consejero, del estudioso del fiqh práctico, se va perfilando.

La aparición del muftí, del alfaquí con capacidad para poder dar respuestas a problemas jurídicos, viene a rellenar este hueco de complemento legislativo del cadiazgo ${ }^{3}$. El muftí aconsejará y aclarará al cadí, será el punto de referencia de las consultas de los miembros de la comunidad, emitirá dictámenes (fetuas) en casos jurídicamente difíciles, sin que se pueda confundir con la noción de juicio, de sentencia (qadà hukm). El muftí será quien dé las reglas especulativo-prácticas, pero no juzga el hecho en sí. Suple la ignorancia del juez, pero se diferencia del mismo en sus funciones.

La legitimación del cargo estar basada en su capacidad para poder ejercitar el iŷtihâd y en la recomendación hecha por el Corán de que toda persona que juzgue, pida consejo, así como en el principio de que la capacidad interpretativa del Corán y la sunna reside teóricamente en la comunidad —en la umma - y en cualquiera de sus miembros: no faltan autores que han resaltado el papel que cumple el muftí como símbolo y personificación de la capacidad de consulta que guarda la comunidad en relación con el poder legislativo, llegándose a considerar al muftí como la encarnación del consensus que debe haber en la comunidad musulmana.

En conclusión, y a manera de resumen, antes de entrar a reseñar someramente la presencia de esta institución jurídica en al-Andalus, podemos definir de una forma global al muftí como un especialista en el fiqh y en la aplicación práctica de éste, que ayuda al juez a resolver las dudas planteadas ante casos concretos y que está al servicio de la comunidad como hombre de consulta, con la misión principal de profundizar en el corpus jurídico ya establecido.

Centrándonos en la España musulmana, y más concretamente, en la época de hegemonía almohade, hay que afirmar, como lo hace 'A.M. Turki, que desde el año 180/796, probable fecha de introducción del malikismo en al-Andalus, y durante los dos siglos de mandato de la

3 Acerca del muftí en al-Andalus, cf. MARTOS QUESADA, J., «Características del muftí en Al-Andalus: contribución al estudio de una institución jurídica hispanomusulmana», en Anaquel de Estudios Arabes, VII (1996), págs. 127-143. 
dinastía omeya, los estudios de jurisprudencia y de metodología jurídica se van a ver peligrosamente decantados y casi monopolizados por una literatura de práctica judicial encaminada a formular soluciones a los numerosos problemas y litigios que aparecían en la vida cotidiana o eran susceptibles de aparecer, escrita en su mayoría por alfaquíes muftíes mālikíes: numerosos prontuarios de masā'il (respuestas jurídicas), de nawãzil (casos que crean jurisprudencia), de fatāwà (consultas jurídicas a muftíes), de ahkām (decisiones judiciales) y de wata $\bar{a}$ 'iq (actas notariales) ven la luz en estos primeros siglos de la España musulmana, todos ellos escritos desde la óptica de la escuela mālikí.

Habrá que esperar a la caída del califato omeya en el año 422/1031, para que la aparición de un sinfín de taifas, de reinos independientes, traiga aparejada una enorme descentralización política, socio-económica y cultural que, a la postre, debía marcar el final del monopolio mālikí en el ámbito jurídico. Es la época en la que tímidamente surgen los estudiosos del hadit y de los ușül al-fiqh, como Ibn 'Abd al-Barr (463/1063) o Ibn Hazm (456/1063), aunque ello no implica que en al-Andalus dejara de interesar la literatura mālikí de «respuestas», como lo prueba la importante recopilación de fetuas llevada a cabo por el abuelo de nuestro Averroes ${ }^{4}$. Sólo con éste, en la época almohade, el interés por el estudio de los uṣull al-fiqh y de las otras escuelas jurídicas no mālikíes, sunníes o no sunníes, conocer un esplendor semejante al que conocen otras regiones del Oriente musulmán y del Magreb de la época.

La domininación almohade en al-Andalus supuso un nuevo fortalecimiento del proceso de berberización de la Península, dirigido ahora por un movimiento nacido como reacción contra la estrechez de las concepciones jurídicas y de los comentarios coránicos almorávides.

No obstante, para un al-Andalus inmerso en un concepto muy restringido de la ortodoxia mālikí, la doctrina y la reforma defendidas por los almohades eran susceptibles de levantar rápidamente sospechas de herejía y heterodoxia que, con el tiempo, - como llegó a demostrarse- llegarían a ser el caldo de cultivo de una resistencia insalvable a la hegemonía almohade.

La pérdida de apoyo popular a los almohades que sucedió a la sensación momentánea de liberación de los abusos almorávides, fue debida en gran parte al marginamiento que sufrieron los alfaquíes y muftíes mālikíes, al reconocimiento indiscriminado de las escuelas zaāirí y šāfíí en detrimento de la tradición mālikí y a la innegable intolerancia religiosa de la que hicieron gala los almohades, la cual hizo realmente difícil la vida de las comunidades no musulmanas. Todo esto desembocar en la crisis definitiva del poder almohade, anunciada en la derrota de las Navas de Tolosa en el año 1212 y evidenciada a partir del año 1220 con su sustitución por los Benimerines en el Magreb.

Con los almohades, los cambios habidos en el ámbito jurídico afectarán más a los aspectos ideológicos que a la misma estructura del ejercicio del Derecho. Este ejercicio sufre una cierta simplificación en comparación con épocas anteriores y el cadí, que vuelve a recuperar la importancia perdida con los almorávides, monopoliza en su persona todo el proceso judicial, a la vez que se da, paralelamente, una pérdida de influencia de los alfaquíes, muftíes y asesores jurídicos en general, siendo numerosos los casos de concentración de actividades judiciales (muftí, cadí,...) en la misma persona.

Por otra parte, en el campo de la legislación, la doctrina almohade se asienta en una serie de principios, que creemos útil reseñar, aunque sea de una forma muy somera; a saber: exclusión del razonamiento y de la apreciación individual (zann) en el establecimiento de la Ley; utilización directa de las fuentes y condena del uso exclusivo de los tratados de furü' o de aplicaciones jurídicas; carácter obligatorio de las formas prohibitiva e imperativa; imposibilidad de restringir a casos particulares lo que est á expresado bajo una forma general; modificación y enriquecimiento posible del fiqh; y, por último, condena de la sola pertenencia a una única 
escuela jurídica. Esta serie de principios llevar a al-Andalus un nuevo estímulo de los estudios de hadīt y de ușull al-fiqh y un nuevo florecer de doctrinas diferentes al malikismo, como la hạamí o la aš' arí.

En este contexto expuesto, la figura del muftí, inevitablemente, acusar estos cambios y sufrir, durante la hegemonía almohade, una evolución con respecto a las fases históricas anteriores, que intentaremos resumir brevemente en cinco puntos.

En primer lugar, su número desciende casi a la mitad en relación con la época almorávide (de 43 muftíes de los que se tenían noticias se pasa a 28 sólamente), es decir, el muftí paga el precio de ser una figura íntimamente ligada al malikismo y su desarrollo, en una etapa en la que esta escuela jurídica sufre la aparición y competencia de otras escuelas y doctrinas jurídicas.

En segundo lugar, a su presencia generalizada en todo el territorio andalusí -y sobre todo en las ciudades y pueblos importantes-, que se detectaba durante los siglos omeyas y de taifas, se contrapone ahora una concentración de los muftíes «profesionales» en Sevilla y el Levante exclusivamente, dato que refleja, incuestionablemente, una pérdida de ésta precisamente mencionada «profesionalidad», al ejercer ahora las labores de muftí, de consejero, en una localidad, el personaje más prestigioso y conocido, tanto por su saber jurídico como por su conducta moral y religiosa, alcanzando su influencia a todas las tierras de los alrededores de su ciudad y excediendo, por tanto, su donación de fetuas el mero ámbito de su lugar de residencia.

En tercer lugar, el arquetipo de muftí como personaje social sufre una interesante variación con respecto a épocas anteriores. A las calificaciones normales aparecidas en sus biografías como «sabio» y «hombre de saber», se les une ahora una serie de adjetivos referentes a su actitud piadosa y religiosa. Asimismo, detectamos una observación, hasta ahora no frecuente, en las noticias biográficas de los mufties: la referida a su popularidad; el buen muftí será, con los almohades, el más conocido por la gente de al-Andalus y sus fetuas las más respetadas, alcanzando este cargo una popularidad que ya tuvo en el Emirato, pero que perdió o se difuminó posteriormente.

En cuarto lugar, desde una visión de su actividad intelectual, la decadencia de la escuela mālikí - tan íntimamente unida a la figura jurídica del muftí- obliga a éstos a adentrarse en el conocimiento de áreas culturales extrajurídicas, como las disciplinas religiosas o las materias relacionadas con el $a d a b$, lo que conlleva, con respecto a la anterior etapa de hegemonía almorávide, un auge mayor de viajes al extranjero para aprender y una mayor dispersión en el número de maestros de Oriente y del Magreb frecuentados por los muftíes de al-Andalus.

Por último, en quinto lugar, se puede observar en los años de dominación almohade un aumento considerable del número de personas que concentran en sí los cargos de muftí, mušăwir o consejero público y cadí, respondiendo de esta manera a la ya mencionada simplificación de la máquina jurídica. Este dato es paralelo al del amplio número de muftíes que ejercen simultáneamente su labor jurídica junto a cargos religiosos, especialmente los de muqrì e imām.

$Y$ ahora, tras este repaso a la figura del muftí y sus peculiaridades en el periodo almohade de al-Andalus, pasaremos a analizar la figura de Averroes como muftí de esta época concreta, dentro del marco de su visión y actividad de la jurisdicción práctica.

\section{LA JURISDICCIÓN PRÁCTICA EN AVERROES: AVERROES COMO MUFTÍ}

Es un hecho innegable que los Banū Rušd de Córdoba constituyen una estirpe con firme tradición jurídica en la España musulmana: todo un linaje de cadíes y muftíes arraigado a lo largo de cinco generaciones de juristas, de alfaquíes, de jurisconsultos.

Y aún cuando su caso no resulte excepcional en la historia de al-Andalus —es probada la existencia de familias con sucesivas generaciones dedicadas al mundo del Derecho-, ofrece, sin embargo, una sugerente peculiaridad, nada desdeñable, como es que, desde el primer Ibn 


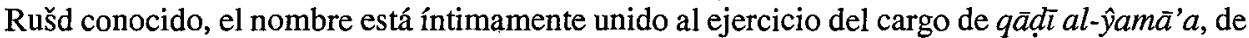
juez mayor de Córdoba, cargo vinculado a la familia y a sus descendientes durante ciento cincuenta años.

Y si a esta hereditaria vocación judicial, que establece un hito de difícil superación, añadimos el prestigio profesional que como muftí alcanzó, dentro y fuera de las fronteras de alAndalus, el abuelo de Averroes, es muy improbable, por no decir imposible, que nuestro Averroes -el nieto- pudiera sustraerse al estudio y ejercicio del fiqh práctico, siguiendo la línea marcada por la actividad jurídica familiar, como parte importante del entramado y la maquinaria judicial que funcionaba en la España musulmana.

La evidente relación de Averroes con el ejercicio del Derecho práctico, no queda probada sólo por los vínculos familiares, sino también por el carácter y perfil intelectual de sus maestros.

Efectivamente, siguiendo a sus fuentes biográficas más directas, y en especial a Ibn alAbbar, podemos extraer de su lista de maestros, al menos seis nombres, cuya relación con el ejercicio activo y la enseñanza de la práctica jurídica está universalmente reconocida.

Estos maestros son, en primer lugar, su padre, Abū-l-Qāsim Aḥmad b. Rušd, muftí y cadí en Córdoba; Abū-1-Qāsim b. Baškuwāl, notario de Córdoba; Abū Marwān b. Masarra, alfaquí cordobés de reconocida fama; Abū Bakr b. Samŷun cadí de Almuñécar; Abū 'Abd Allāh alMazrī, alfaquí de origen siciliano que le dio la $i \hat{y} \bar{a} z a ; A b \bar{u}$ Marwān al-Balansī, prestigioso muftí de Valencia; y Abū Bakr b. Rizk, alfaquí de la zona de Almería.

Todos ellos le iniciaron en el conocimiento y profundización, no ya sólo del fiqh práctico, sino también del pensamiento jurídico teórico, lo que le permitió a Averroes ejercer una actividad jurídica práctica propia, tanto de alfaquí, como de muftí y cadí, que queda reflejada en los datos biográficos de sus fuentes, tanto directas como indirectas.

De las siete fuentes directas, de los siete elencos biográficos que se hacen eco de su vida, sólo al-Ḍabbī, Ibn 'Arabī e Ibn al-Abbār nos dan cumplida cuenta de su actividad en el fiqh práctico, mientras Ibn Abī Ușaybi'a, al-Marrākuši, Ibn Sa 'īd al-Magribī y al-Anșarīi ${ }^{6}$, se centran más en sus facetas filosófica o médica, o bien en datos de su biografía como su caída en desgracia ante el sultán almohade. En cuanto a sus fuentes indirectas (al-Dahabī, al-Șafadī, alŠātibi, al-Maqqarī y Hậŷŷ̀̃ Jalifa) ${ }^{7}$, si exceptuamos a al-Maqqarī y las citas de Hāâŷȳi Jalifa, los datos ofrecidos por el resto de los autores es desconsolador, con abundancia de errores.

Como muftí, por la información que se desprende de al-Dabbī e Ibn 'Arabī y certificada expresamente por Ibn al-Abbār, sabemos que ejerció casi con toda seguridad en Sevilla, Córdoba y Marraquech, muy probablemente antes de su nombramiento como cadí en Sevilla.

Su actividad como cadí se encuentra, obviamente, más documentada y, así, nos consta en sus fuentes biográficas que fue nombrado cadí en la ciudad de Sevilla en el año 565/1169 y cadí mayor, qãặ al-ŷamā'a de Córdoba en el año 576/1180 6́ 578/1182, ejerciendo probablemente asimismo el cadiazgo en Marraquech entre estos años.

El nombramiento de qậ̣̂ al-y̧ama 'a en Córdoba implicaba rango visirial y tenía un triple carácter religioso, político y jurídico. Era el encargado de dirigir la oración pública los viernes en la mezquita mayor, a la vez que desempeñaba otras funciones canónicas. Por otra parte, era miembro del consejo real y se le encomendaban delicadas misiones diplomáticas. En el ám-

5 AL-ḌABBI, Bugya, ed. Codera, BAH, n 3, Madrid, 1885, pág. 44; IBN 'ARABĪ, Futuh, ed. El Cairo, 1972, vl. III, pág. 372; IBN AL-ABBĀR, Takmila, ed. Codera, BAH, nº s. 4-5, Madrid, 1886, vl. II, pág. 269.

6 IBN ABĪ UȘAYBI'A, tabaqat, ed. El Cairo, 1889, vl. II, págs. 75-78; AL-MARRĀKUŠ̀̃ , MU' 'ŶIB, ed. Leiden, 1881, págs. 174-1755 y 225-226; IBN SA'ĨD AL-MAGRIBİ, Mugrib, ed. Beirut, 1964, vl. I, $2^{\circ}$; AL-ANȘARI, Dayl, ed. Beirut, 1973, vl. VI, págs. 21-31.

7 AL-DAHABĪ, Ta'rij, ed. El Cairo a partir de 1947, Apéndice IV, págs. 456-462; AL-ȘAFADĪ, Wafi, ed. Estambul, 1949, vl. II, págs. 114-115; AL-Š $\bar{A}$ TIBI, 'Uqud, manuscrito París, BN, 1545/9; AL-MAQQARI, Nafh, ed. Leyden, 1855-1861, 2 vls; Hฺ̄ÂYY $\hat{Y} \bar{I}$ JALİFA, Kašf, ed. Estambul, 1941. 
bito jurídico le correspondía, por delegación soberana, el enjuiciamiento en ultima instancia de los asuntos civiles en los que uno de los litigantes al menos fuera musulmán y tuvieran por objeto los bienes de manos muertas, divorcios, declaraciones de incapacidad, testamentos, particiones, sucesión intestada, administración del patrimonio de los ausentes, huérfanos e incapacitados y el juzgar todos aquellos litigios en que fuera parte el Estado, así como ciertos delitos religiosos. En suma, era la cabeza visible de la Administración de Justicia, al que le estaban subordinados los otros jueces con potestad para dictar sentencia.

Hay que decir que toda su actividad jurídica práctica, tanto sus fetuas, sus respuestas jurídicas, como sus sentencias, están basadas en algo más que en dar una solución inmediata, más o menos prototípica, a un litigio presente; sus actuaciones como ejercente del Derecho práctico desprenden un principio de actuación, unas posiciones teóricas, que podemos sintetizar en cuatro:

En primer lugar, ante el hecho de que la matización de las sentencias variaba tanto en la práctica, que era casi imposible establecer una jurisprudencia común, Averroes adopta como primera precaución universalizar el $i \hat{y} t i h \bar{a} d$, el esfuerzo intelectual, el uso del razonamiento individual, para intentar una mayor objetividad, racionalidad y sistematización; conviene abandonar los casos raros, inhabituales y menores para centrarse en los fundamentos ( $u s ̦ u l)$, trazar las líneas generales y señalar las cuestiones básicas o madres de las que nacen todas las demás.

En segundo lugar, en el uso del qiyās, del razonamiento por analogía, del razonamiento inductivo, Averroes distinguía entre el qiȳas yaqīn̄i o absoluto y el qiyās zannī o probable. Este último debe desterrarse como fundamento en los problemas jurídicos muy dudosos; de la misma forma, el uso del qiyās sólo puede aplicarse en los casos rectamente establecidos, siendo ilícito su uso para los erróneos, pudiendo pasarse de una expresión particular a su sentido general, siempre que se trate del tránsito de algo concreto y tomado como tal a otro particular, siempre que se apoye en un sentido casual o de similitud.

En tercer lugar, el recurso al iŷma $\bar{a}^{*}$, al consenso es complejo, debiendo tenerse en cuenta que sólo tiene un carácter de alta probabilidad en tanto se apoya en la coincidencia interpretativa.

Por último, en cuarto lugar, Averroes apuesta por buscar la solución o soluciones propuestas, tanto por la escuela propia como por las otras escuelas, cuando son aceptables, intentando explicar la causa de las discordancias. Ya que hay textos que admiten más de una interpretación y tradiciones sobre cuya autenticidad caben dudas, hay que insistir en la búsqueda de normas lógicas, en el gusto por la racionalidad y en la buena marcha dialéctica, teniendo en cuenta, en todo caso, la imposibilidad de subordinar el razonamiento racional a las necesidades de la dialéctica.

Y para finalizar, no podemos dejar de citar algunas de las soluciones personales que, tanto en su calidad de muftí como de cadí, dio Averroes a la compleja casuística del fiqh islámico y que se encuentran contenidas en su obra Bidāya. Como ejemplos, tomaremos algunas referidas al Derecho de Familia (matrimonio, repudio, tutela legal y sucesiones), tomadas de los trabajos de R. Brunschvig y M. Cruz Hernández.

En cuanto al matrimonio, desde una perspectiva liberal, limita el uso de la obligación de contraer matrimonio por imposición familiar a los impúberes, aun en el supuesto de que la muchacha hubiera dejado de ser virgen; considera Averroes que la mujer que ha consumado su matrimonio, tras la disolución de éste, no necesita de un tutor matrimonial; sostiene que las capitulaciones matrimoniales en las que el marido se compromete a no tomar una segunda esposa o a no cambiar de residencia, obligan; igualmente, mantiene el valor de la palabra de la mujer cuando est en desacuerdo sobre la entrega de la dote.

8 BRUNSCHVIG, R., obra citada, págs. 189-199; CRUZ HERNÁNDEZ, M. Vida, obra, pensamiento, influencia de Abū-l-Walid Muhammad Ibn Ruṣd (Averroes), $2^{\mathrm{a}}$ ed., Córdoba, 1997. 
Respecto al repudio, no admite un triple repudio simultáneo, pues el Islam admite hasta cuatro esposas al mismo tiempo; tiende a igualar a los esclavos y al hombre libre en esta materia, y los derechos de la esclava madre con los de las esposas en dicho caso. Se opone a que la esposa que se niega a jurar sobre su presunta infidelidad sea azotada o lapidada, debiéndosele mantener encarcelada hasta que preste dicho juramento.

Sobre la tutela legal, no admite Averroes el plazo de demora que los mālikíes imponían a la mujer tras el matrimonio para poder disponer de sus bienes, ni la del muchacho tras de la pubertad a los mismos efectos.

Y en cuanto a las sucesiones, no rechaza los derechos a la sucesión de los parientes hembras o de los deudos políticos; da preferencia al abuelo paterno sobre los hermanos del causante; también se inclina por el respeto a los derechos del hijo, respecto de los bienes de la madre, en caso de ser adulterino o de haber sido anatemizada la madre.

\section{CONCLUSIÓN}

Finalmente, y como conclusión, podemos apuntar dos aspectos, que no deberían perderse de vista en futuras investigaciones averroístas.

En primer lugar, poner de relieve que el estudio de la actividad, obra y pensamiento jurídicos de Averroes, adolece de una cierta escasez de trabajos, si se compara con los existentes sobre el Averroes filósofo o científico; hecho incomprensible si tenemos en cuenta que las ideas y la obra jurídica del mismo supusieron una revolución en el pensamiento mālikí hegemónico en todo el Derecho de la España musulmana.

En segundo lugar, la visión y el análisis del Averroes jurista, no debe sobredimensionar excesivamente sus valores y aportaciones teóricas, de tal modo que acabe perdiéndose de vista su actividad en el fiqh práctico, a la cual dedicó gran parte de su vida y tiene su reflejo de una forma evidente a lo largo de sus escritos sobre jurisprudencia.

Juan Martos

Departamento de Árabe Universidad Complutense E-28040 Madrid

E-mail: juanmartos@telefonica.es 